\title{
Appendix. Molecular Hydrogen in the Interstellar Gas
}

\author{
E. E. SALPETER \\ Laboratory of Nuclear Studies, Physics Department, and Center for Radiophysics and \\ Space Research, Cornell University, Ithaca, N.Y., U.S.A.
}

There are two levels of interest in the abundance of molecular hydrogen in the interstellar gas. (i) Even a low abundance can enhance the cooling rate at a few hundred K. (ii) Densities comparable with or larger than those of atomic hydrogen could affect the dynamics in the galactic disk, if widespread, or at least affect star formation, if restricted to isolated large clouds.

Extensive calculations of growth rates were carried out some time ago by Gould and Salpeter (1963) and by Gould et al. (1963), and the situation as of a few years ago can be summarized as follows (Field et al., 1966). At densities normal for ordinary interstellar clouds $\left(n_{\mathrm{H}} \approx 10 \mathrm{~cm}^{-3}\right)$ a hydrogen atom strikes the surface of a dust grain about every $10^{7} \mathrm{yr}$. For grain temperatures $T_{\text {gra in }}$ below about $20 \mathrm{~K}$ the recombination efficiency $\gamma$ (probability of an $\mathrm{H}$-atom to end up in a molecule after hitting the grain surface) was thought to be quite high, $\gamma \approx \frac{1}{3}$. The threshold for photodissociation of $\mathrm{H}_{2}$ was thought to be beyond the Lyman continuum, in which case molecular hydrogen would break up only when a cloud came near an OB star. The net result was the belief that molecular hydrogen might generally be about as abundant as atomic hydrogen and much more abundant in dense clouds.

In recent years, ideas of high $\mathrm{H}_{2}$-abundances received two separate blows. (i) The inclusion of quantum mechanical zero-point energy lowers the adsorption binding energy for a hydrogen atom on a perfect crystal surface (Knaap et al., 1966). It was concluded that the atoms would evaporate instead of recombine unless $T_{\text {grain }}<8 \mathrm{~K}$. Pure graphite grains would have temperatures well above $20 \mathrm{~K}$ and the recombination efficiency $\gamma$ would be very low. (ii) Stecher and Williams (1967) found that about 10 per cent of excitations of $\mathrm{H}_{2}$ to a set of discrete levels, brought about by photons with $\lambda>912 \AA$, are followed by dissociation. The lifetime of a hydrogen molecule in normal interstellar space (HI-region) against this radiative destruction is only about $10^{3} \mathrm{yr}$. One might then have expected the $\mathrm{H}_{2}$-abundance to be quite negligible throughout interstellar space.

More recently still, the first difficulty was overcome in two ways: (a) Grains are now thought to contain enough impurities and/or dielectric mantles to radiate fairly efficiently in the infrared, so that grain temperatures well below $15 \mathrm{~K}$ are now expected. (b) Surface defects of various types on realistic grains are expected to provide some sites with increased adsorption binding energy. Estimates indicate good recombination efficiency as long as $T_{\text {grain }} \lesssim 25 \mathrm{~K}$ (Hollenbach, 1969; Hollenbach and Salpeter, 1970).

The second difficulty persists under normal interstellar conditions: in an 'ordinary' interstellar cloud $\left(n_{\mathbf{H}} \approx 10\right.$ to $30 \mathrm{~cm}^{-3}$, optical depth in the visible of $\tau_{\mathrm{vis}} \lesssim 0.2$ ), the 
molecular abundance of hydrogen is only about $10^{-5}$ times the atomic abundance. $\mathrm{H}_{2}$ is therefore unlikely to play a dominant role in the dynamics of the galactic disk as a whole. However, the situation is radically different in the denser clouds which presumably are the precursors of star formation. Since the relevant photodisintegration is produced by line-radiation, the interior of a dense enough cloud can be shielded from this line-radiation by self-absorption in $\mathrm{H}_{2}$. The $\mathrm{H}_{2}$ is replenished by continuous recombination in the outer layers of the cloud and the attenuation of the radiation is also helped by the usual grain absorption. Estimates show that hydrogen should be mainly molecular in any cloud with $n_{\mathrm{H}} \gtrsim 100 \mathrm{~cm}^{-3}$ and $\tau_{\mathrm{vis}} \gtrsim 0.5$ (Hollenbach 1969; Solomon, to be published). One direct observation (Werner and Harwit, 1968) and some observed anticorrelation (Garzoli and Varsavsky, 1966; Heiles, 1969) between atomic hydrogen and dust-density in dense clouds seem to corroborate these estimates. *

\section{References}

Field, G. B., Somerville, W., and Dressler, K.: 1966, Ann. Rev. Astron. Astrophys. 4, 207.

Garzoli, S. L. and Varsavsky, C.: 1966, Astrophys. J. 145, 79.

Gould, R. J., Gold, T., and Salpeter, E.: 1963, Astrophys. J. 138, 408.

Gould, R. J. and Salpeter, E. E.: 1963, Astrophys. J. 138, 393.

Heiles, C.: 1969, Astrophys. J. 156, 493.

Hollenbach, D. J.: 1969, Ph.D. thesis, Cornell University, Ithaca.

Hollenbach, D. J. and Salpeter, E. E.: 1970, Astrophys. J. (in press).

Knaap, H., v. d. Meijdenberg, C. J. N., Beenakker, J., and Van de Hulst, H. C.: 1966, Bull. Astron. Inst. Netherl. 18, 256.

Solomon, P. M. (to be published).

Stecher, T. P. and Williams, D. A.: 1967, Astrophys. J. Lett. 149, L29.

Werner, M. W. and Harwit, M.: 1968, Astrophys. J. 154, 881.

* (Note added in proof.) Carruthers (1970, Astrophys. J. Lett. 161, L 81) announced the detection of interstellar $\mathrm{H}_{2}$ in the spectrum of $\chi$ Per. (Ed.) 